\title{
Temporär genutzte Räume von berufsbedingt multilokal Lebenden. Herausforderungen und Potenziale für betriebliche und regionale Entwicklungen in ländlichen Räumen Niedersachsens
}

\section{Temporarily used places of multi-local living employees. Challenges and potentials for operational and regional developments in rural areas of Lower Saxony}

https://doi.org/10.2478/rara-2019-0045

Eingegangen: 28. September 2018; Angenommen: 1. Juli 2019

Kurzfassung: Aufgrund von Globalisierungsprozessen in Zusammenhang mit zunehmender Individualisierung und Pluralisierung von Lebensstilen sowie flexibilisierten Arbeitswelten nimmt räumliche Mobilität zu. Dabei werden multilokale Lebensweisen, also Lebensführungen an mehreren Orten und über mehrere Orte hinweg, immer bedeutsamer. In diesem Beitrag werden erste Ergebnisse einer Analyse berufsbedingt multilokaler Lebensweisen in ländlichen Räumen vorgestellt. Ziel des Beitrages ist es, Herausforderungen und Potenziale multilokaler Lebensweisen für betriebliche und regionale Entwicklungen aufzuzeigen sowie damit zusammenhängende mögliche Handlungsfelder für die politisch-planerische und unternehmerische Praxis zu formulieren. Mithilfe von qualitativen Forschungsmethoden wurde eine Fallstudienanalyse im niedersächsischen Landkreis Diepholz durchgeführt. Der Beitrag diskutiert zunächst die Beweggründe und Umstände, die dazu führen, dass Beschäftigte an mehreren Orten leben (müssen). Danach wird die Gestaltung des Altags- und Berufslebens der mehrörtig lebenden Personen in temporären, das heißt zeitweise bewohnten Räumen, erläutert. Des Weiteren wird gezeigt, welche betrieblichen und regionalen Rahmenbedingungen und Strukturen diese Lebensweisen beeinflussen und welche Bedeutung berufsbedingte Multilokalität für betriebliche und regionale Entwicklungen haben kann.

Schlüsselwörter: Multilokalität, ländliche Räume, allägliche Lebensführung, Betriebe, Regionalentwicklung, Niedersachsen, Diepholz

Abstract: Due to globalization processes in connection with increasing individualization and pluralization of lifestyles as well as flexible working environments, spatial mobility is increasing. A multi-local way of life, thus living in several places, is becoming more and more important. This paper presents the first results of an analysis of occupational

\footnotetext{
*Corresponding author: Lena Greinke, Leibniz Universität Hannover, Institut für Umweltplanung, Herrenhäuser Straße 2, 30419 Hannover, Deutschland, E-mail: greinke@umwelt.uni-hannover.de

Dr. Nicola Hilti, Fachhochschule St. Gallen, Institut für Soziale Arbeit und Räume, Rosenbergstraße 59, 9001 St. Gallen, Schweiz

2 Open Access. (c) 2020 Lena Greinke, Nicola Hilti, published by Sciendo. (cc)Br-NC-ND This work is licensed under the Creative Commons Attribution-NonCommercial-NoDerivatives 4.0 License.
} 
multi-local lifestyles in rural areas. This contribution aims to identify challenges and potentials of multi-local lifestyles for operational and regional developments and to formulate associated possible fields of action for political-planning practice and entrepreneurial practice. With the help of qualitative methods, a case study analysis was carried out in the district Diepholz in Lower Saxony (Germany). The article first discusses the motives and circumstances that ensure that employees (have to) live in several places. Afterwards, the organization of everyday life and working life of the persons living in temporarily used places is explained. Furthermore, it will be shown which operational and regional framework conditions and structures influence these lifestyles, as well as the significance of job-related multi-locality for operational and regional developments.

Keywords: Mutli-locality, Rural areas, Everyday life, Companies, Regional development, Lower Saxony, Diepholz

\section{Berufsbedingte Multilokalität und seine Bedeutung}

Räumliche Mobilität spielt in der heutigen Zeit eine zentrale Rolle. Eine zunehmend bedeutsame Ausprägung mobiler Lebensführungen aufgrund individualisierter und pluralisierter Lebensstile sowie flexibilisierter Arbeitswelten ist Multilokalität (Dittrich-Wesbuer/Föbker 2013: 391; ARL 2016: 1), das heißt das Wohnen und Leben an mehreren Orten in einem mehr oder weniger regelmäßigen Rhythmus. Die Motive, Beweggründe und Ausprägungsformen sind dabei äußerst vielfältig (Hilti 2013: 32; Seebacher 2013: 12). Multilokale Lebensweisen sind historisch betrachtet nichts Neues. Im Zuge zunehmender globaler und zugleich räumlicher Arbeitsteilung (vgl. Hesse/Scheiner 2007: 138) verstärken sie sich jedoch, differenzieren sich aus und sind in allen gesellschaftlichen Schichten und Milieus verankert (Rolshoven 2007: 160 ff.; Hilti 2009: 77; Schier 2009: 57; Weiske/Petzold/ Zierold 2009: 67).

In diesem Beitrag steht die berufsbedingte Multilokalität in ländlichen Räumen Niedersachsens im Mittelpunkt. Ländliche Räume sind oft komplexer als in der räumlichen Planung angenommen (Altrock/Güntner/ Huning et al. 2005: 7), denn sie sind von komplexen Wandel- und Veränderungsprozessen betroffen: Neben dem demographischen Wandel zählen auch klimatische sowie wirtschaftsstrukturelle Prozesse dazu, die unmittelbar auch Wohn- und Arbeitsverhältnisse, Freizeit- und Mobilitätsverhalten der Menschen beeinflussen (NHB/ALR 2014: 3). Restrukturierungsprozesse am Arbeitsmarkt (vgl. Reuschke 2010: 287) und neue Unternehmensstrukturen begünstigen zudem multilokale Lebensformen, beispielsweise unternehmensinterne Delegationen (internationaler) Mehrbetriebsunternehmen an verschiedenen Standorten, häufig wechselnde Arbeitsorte oder Arbeitnehmerüberlassungen in Form von temporärer Leiharbeit. Diese „Erosion des Normal- arbeitsverhältnisses“ (Sturm/Meyer 2009: 22) führt zu erhöhten Mobilitätsanforderungen.

Vor dem Hintergrund des skizzierten gesellschaftlichen Wandels multilokalisieren sich Menschen zunehmend (Petzold 2013). In ihren Praktiken der Lebensführung konstituieren sie stets aufs Neue Räume, die sie jeweils in spezifischen raum-zeitlichen Mustern nutzen. Das ,Herstellen' dieser temporär genutzten Räume erfolgt über die physische (aber auch virtuelle) Mobilität der Menschen, aber ebenso über die Verankerung an den jeweiligen Orten. Ausschlaggebend für die Lebensführung und aus der Perspektive der multilokal Lebenden sind demnach nicht die territorialen Grenzen, sondern die ,gemachten' Räume.

In diesem Beitrag wird von einem breiten Verständnis des multilokalen Wohnens ${ }^{1}$ ausgegangen, sodass auch Personen, die nicht an allen ihren Wohnorten melderechtlich erfasst sind, mit eingeschlossen sind (vgl. Lange 2018). Dadurch ist es möglich, auch „verdeckte Multilokalität" oder "Kryptomultilokalität“ (Weichhart/ Rumpolt 2015: 17 f.) (z. B. regelmäßige Übernachtungen bei Freunden oder in Hotels) zu erfassen. Nicht berücksichtigt hingegen wird die Palette an eher freizeitbezogenen und partnerschaftlich bedingten multilokalen Lebensführungen. Die Forschung hat sich bislang eher auf städtische Gebiete konzentriert (Dannenberg/Lang/ Lehmann 2010: 55), mit wenigen Ausnahmen (z. B. Lange 2018). Allerdings befinden sich temporäre Wohnorte sowohl in städtischen wie auch in ländlichen Gebieten (Greinke/Lange/Othengrafen 2017). Ländliche Gebiete sind in diesem Zusammenhang von großer Bedeutung, übernehmen sie doch - entgegen der Zuschreibungen als Orte der Agrarproduktion, der ökologischen und Erholungsfunktionen - zunehmend auch Funktionen als Standorte beispielsweise für Gewerbe und Dienst-

1 Multilokalität und multilokales Wohnen werden in diesem Beitrag synonym verwendet. 
leistungen (Dannenberg/Lang/Lehmann 2010: 56), und unterscheiden sich in ihren sozioökonomischen Strukturen von urbanen Räumen (BMVBS/BBSR 2009: 4). In zahlreichen ländlichen Gebieten haben sich Unternehmen angesiedelt (vgl. BMEL 2016), von denen aufgrund ihrer Betriebsstrukturen mit z. B. unternehmensinternen Delegationen der Beschäftigten an verschiedene Standorte Ballungen von mehrörtig lebenden Beschäftigten zu erwarten sind. Vor diesem Hintergrund stellen sich folgende Fragen:

- Welche Beweggründe und Umstände führen dazu, dass Beschäftigte an mehreren Orten leben (müssen) und wie gestalten diese Personen ihr Alltags- und Berufsleben in den von innen temporär genutzten Räumen?

- Welche betrieblichen und regionalen Rahmenbedingungen und Strukturen beeinflussen multilokale Lebensweisen in ländlichen Gebieten?

- Welche Bedeutung kann diese berufsbedingte Multilokalität für betriebliche und regionale Entwicklungen in ländlichen Gebieten haben und welche Handlungsfelder für die politisch-planerische und unternehmerische Praxis lassen sich daraus ableiten?

Diesen Fragen geht der vorliegende Beitrag nach mit dem Ziel, die Herausforderungen und Chancen aufzuzeigen, die sich aufgrund der mehrörtig lebenden Beschäftigten für die Betriebe und die Regionalentwicklung in ländlichen Räumen Niedersachsens ergeben. Der Beitrag stellt Zwischenergebnisse eines Dissertationsprojektes zur Diskussion, welches im Rahmen des Forschungsprojekts TempALand ${ }^{2}$ durchgeführt wird. Dazu werden zunächst der Forschungsstand zu (berufsbedingter) Multilokalität (Kapitel 2) und danach das methodische Vorgehen im Rahmen einer Fallstudienanalyse im niedersächsischen Untersuchungsgebiet, dem Landkreis Diepholz in Deutschland (Kapitel 3), vorgestellt. Anschließend werden die Ergebnisse der Analyse der mehrörtig lebenden Beschäftigten darge-

2 Das dreijährige Forschungsprojekt „Temporäre An- und Abwesenheiten und deren Auswirkungen auf Land und Gesellschaft" (www.tempaland.de) wird mit Mitteln des Bundesministeriums für Bildung und Forschung innerhalb der Fördermaßnahme „Kommunen innovativ“ von 2016 bis 2019 gefördert. Projektpartner und -beteiligte sind das Institut für Umweltplanung der Leibniz Universität Hannover (Projektleitung), der Landkreis Diepholz (Fachdienst Kreisentwicklung), fünf kommunale Praxispartner im Landkreis und die beiden Planungsbüros Gertz Gutsche Rümenapp - Stadtentwicklung und Mobilität sowie proloco - Stadt und Region, Planung und Entwicklung. stellt und die Bedeutung dieser berufsbedingten Multilokalität für betriebliche und regionale Entwicklungen erläutert (Kapitel 4). Abschließend werden erste Handlungsfelder für betriebliche und regionale Entwicklungen in ländlichen Räumen Niedersachsens aufgezeigt und ein Ausblick auf weitere mögliche Entwicklungen und Forschungsthemen gegeben (Kapitel 5).

\section{Forschungsstand zu berufsbedingter Multilokalität}

Die jüngere Multilokalitätsforschung hat ihre Anfänge in den 2000er-Jahren. Ihre kulturwissenschaftlichen Wurzeln gehen jedoch bis in die 1970er-Jahre zurück. Das Phänomen wurde bislang breit und interdisziplinär erforscht. Daher lässt sich die Multilokalitätsforschung am besten entlang verschiedener interdisziplinärer Forschungsfelder darstellen.

\subsection{Mobilitätsforschung}

Die Mobilitätsforschung befasst sich mit vielen Facetten von Mobilität und dabei mitunter - meist implizit - auch mit Multilokalität, die als hybride Querschnittsmaterie zu den drei großen Mobilitätsthemen der globalisierten Spätmoderne betrachtet wird: „... the phenomenon of dual residency straddles the areas of residential mobility, travel and daily mobility" (Kaufmann 2002: 36).

In der Erforschung des Phänomens interessieren spezifische Aspekte oder Typen, etwa die Beweggründe und Ausprägungen berufsbedingten multilokalen Wohnens (Collmer/Kümmel 2005) sowie dessen Folgen für verschiedene Lebensbereiche, wie das Familienleben (Schier 2009), soziale Netzwerke (Larsen/Axhausen/Urry 2006), die psychische Gesundheit (Schneider/ Limmer/Ruckdeschel 2002) oder die Motilität im Sinne des (ungleich verteilten) Potenzials zur Mobilität (Viry/ Kaufmann/Widmer 2008). Aber auch bestimmte Berufsgruppen wie z. B. Piloten (Huchler 2013), Beschäftigte in der Erdgas- und Erdölindustrie (Saxinger 2016) oder der Kreativwirtschaft (Nadler 2014) stehen im Fokus einiger Untersuchungen.

Ein Strang der jüngeren Mobilitätsforschung - die Mobilities Studies - befasst sich mit der Bewegung und Beweglichkeit von Menschen, Gütern, Informationen und Ideen als konstitutive Elemente der spätmodernen Gesellschaft (Urry 2007) und richtet dabei die Perspektive zunehmend weg von der einseitigen Betonung des 
Mobilen und hin zum Spannungsfeld von Mobilität und Verankerung (Sheller 2011; Meier/Frank 2016).

\subsection{Migrations-, Transnationalismus- und Entwicklungsforschung}

Die Forschung zu Migration und Transnationalismus untersucht unter anderem spezifische Formen von Multilokalität, insbesondere im Bereich Transmigration (Pendeln zwischen Wohnorten in kulturell unterschiedlichen Zusammenhängen) und Amenity Migration (Wohlstandsmigration, vorwiegend freizeitorientiert). Auch hier werden seit einiger Zeit vermehrt die sozialen, kulturellen und ökonomischen Wechselbeziehungen zwischen den verschiedenen Aufenthaltsorten als konstitutiv für Migrationsprozesse in den Mittelpunkt gerückt (Albrow 1997; Pries 1998). Konzepte mit einem Fokus auf transnationale oder multilokale Haushalte ermöglichen ein besseres Verständnis etwa von Verstädterungsprozessen und familialen Dynamiken im Kontext von Migration (Schmidt-Kallert 2009; Steinbrink 2009).

Auch in der Entwicklungsforschung kommt den Wechselbeziehungen und der Multilokalität wachsende Aufmerksamkeit zu (de Haan/Zoomers 2003): Die Sicherung der Existenz hängt in vielen Haushalten in Ländern des globalen Südens von der Mobilität einzelner Familienmitglieder ab. Die Ausprägungen und Folgen solcher Lebensführungen werden anhand von Konzepten wie livelihood (Sicherstellung der Existenzgrundlagen einer Gesellschaft) und vulnerability (Verwundbarkeit von Personen und Gruppen im Kontext der Entwicklungsforschung) beschrieben (Schmidt-Kallert 2009; Dick/ Reuschke 2012). Die Multilokalitätsforschung hat hieran angeknüpft und ihre Protagonistinnen/Protagonisten schlugen vor, diese Konzepte auf sämtliche Formen multilokalen Daseins anzuwenden (Weichhart 2015).

\subsection{Tourismus- und Zweitwohnsitzforschung}

Zweitwohnsitze sind ein Teilphänomen der Multilokalität, welche hauptsächlich in Zusammenhang mit Tourismus sowie seinen wirtschaftlichen und sozialen Auswirkungen erforscht werden (Gallent/Tewdwr-Jones 2000; Hall/ Müller 2004), wie auch die Freizeitmobilität und Verkehrsverhalten (Fuhrer/Kaiser 1994). Die Betrachtung touristischer Praktiken fokussiert vornehmlich den Ort des zweiten Wohnsitzes, den touristischen Zielort. Die große Mehrheit der Zweitwohnsitzforschung konzent- riert sich auf ländliche bzw. alpine Regionen. Eine frühe Ausnahme hierzu bildet eine Untersuchung zu berufsbedingten Zweitwohnsitzen in fünf Schweizer Städten (Odermatt 1990).

Darüber hinaus wird in der subjektorientierten Multilokalitätsforschung die Hierarchisierung der Wohnsitze über Bezeichnungen wie Erst- und Zweit- oder Hauptund Nebenwohnsitz kritisch reflektiert, da sich diese Hierarchien häufig nicht in den Praktiken und Bedeutungen der Wohnsitze widerspiegeln (Hilti 2013). Auch die oftmals implizierte funktionale Trennung etwa zwischen dem Ort der Arbeit und dem Ort der Freizeit wird hinterfragt, da diese häufig nicht den Lebensrealitäten entspricht (Wood/Hilti/Kramer et al. 2015).

\subsection{Wohn-, Haushalts- und Familienforschung}

Die Wohn-, Haushalts- und Familienforschung ist lange Zeit einer eher wenig mobilen Vorstellung ihrer Untersuchungsgegenstände gefolgt: Das Wohnen wurde als statisch und an einem Ort stattfindend konzipiert (Weiske/Petzold/Zierold 2009; Schier/Hilti/Schad et al. 2015). Demgegenüber steht ein Verständnis von Praktiken dynamischer, sich über mehrere Orte hinweg erstreckender Haushalte und Familien (Dittrich-Wesbuer/Föbker/Sturm 2015). Multilokale Haushalte werden dann zu zentralen gesellschaftlichen Akteuren (SchmidtKallert 2009; Steinbrink 2009; Weiske/Petzold/Zierold 2009).

In der Familienforschung steht seit Längerem das "Leitbild der Normalfamilie“ (Beck 2008: 305), dem unter anderem eine monolokale Familie entspricht, in der Kritik. Stattdessen widmet sie sich vermehrt dem strukturellen Wandel von Familien, zu welchem auch multilokale Verflechtungen von Menschen und Orten zählen. Im Forschungsinteresse stehen dann etwa multilokal lebende Kinder aus sogenannten Nachtrennungsfamilien oder von berufsbedingt temporär abwesenden Elternteilen (Schier 2009; Schier 2010) sowie erwerbstätige Mütter und Väter der Film- und Fernsehproduktion und aus dem Einzelhandel (Jurczyk/Schier/Szymenderski et al. 2009).

\subsection{Raumwissenschaftliche Forschung}

Die räumlichen und zeitlichen Dimensionen von Multilokalität wurden bislang mehr oder weniger explizit stets berücksichtigt (Weichhart 2009; Wood/Hilti/Kramer et al. 2015). Eine wichtige und bislang kaum geklärte Frage 
ist jedoch diejenige nach den räumlichen und raumplanerischen Auswirkungen dieser spezifischen Art der Lebensführung. Dabei geht es unter anderem darum, was das Phänomen der Multilokalität für den Wohnungsmarkt, für die Stadtentwicklung, die Stadtpolitik und das bürgerschaftliche Engagement bedeuten kann (Leubert 2013; Dittrich-Wesbuer/Plöger 2013; Nadler 2014; ARL 2016; Dittrich-Wesbuer 2016; Greinke/Lange/Othengrafen 2018; Lange 2018).

Ein Teil dieser explizit raumwissenschaftlich ausgerichteten Forschung, darunter insbesondere auch die in diesem Beitrag auszugsweise vorgestellte laufende Studie, nimmt wichtige Facetten auf, die bisher unterbelichtet geblieben sind. Bislang wurde das Wohnen an mehreren Orten vor allem im städtischen Kontext oder ohne spezifische Sortierung von Raumtypen untersucht, wohingegen hier nun ländliche Räume im Mittelpunkt stehen. Die bisherige Forschung hat sich großteils auf die Subjektperspektive konzentriert, auf die lebensweltliche Bedeutung einer solchen multilokalen Lebensführung. Inzwischen werden auch andere wichtige Perspektiven einbezogen, etwa aus Politik und Wirtschaft.

\section{Methodisches Vorgehen}

In diesem Beitrag werden mehrörtige Lebensweisen von Beschäftigten in ländlichen Räumen und deren Auswirkungen auf betriebliche und regionale Entwicklungen mithilfe von quantitativen Methoden untersucht. Auf der Basis einer Grundlagenrecherche zur Thematik der Multilokalität wurde eine Fallstudienanalyse im Untersuchungsgebiet, dem niedersächsischen Landkreis Diepholz, durchgeführt. Vor allem der südliche Teil des Landkreises wurde anhand einer zweistufigen sozioräumlichen Analyse auf Kreisebene in Niedersachsen nach Lange (2018) als ländlich geprägter Raum mit dort ansässigen, zumeist international tätigen Unternehmen identifiziert. Dort ist mit berufsbedingten phasenweisen An- und Abwesenheiten der Bewohnerinnen/Bewohner zu rechnen (Lange 2018: 60, 176).

\subsection{Der Landkreis Diepholz als Untersuchungsgebiet}

Der niedersächsische Landkreis Diepholz, bestehend aus acht Einheitsgemeinden und sieben Samtgemeinden, liegt im Dreieck zwischen der Hansestadt Bremen im Norden, der Landeshauptstadt Hannover im Südos- ten und der Stadt Osnabrück im Südwesten. Der Landkreis ist im Norden aufgrund der Nähe zur Stadt Bremen durch wirtschaftlich eher starke (urbane) Räume (z. B. die Gemeinden Stuhr und Wehye und die Stadt Syke) gekennzeichnet. Aus diesem Grund wird der Fokus in dieser Untersuchung auf den ländlich geprägten südlichen Teil des Landkreises mit der Stadt Bassum und der Stadt Diepholz, der Gemeinde Wagenfeld und den Samtgemeinden Altes Amt Lemförde, Barnstorf, Bruchhausen-Vilsen, Kirchdorf und Rehden gelegt.

Ähnlich wie andere ländlich geprägte Räume Deutschlands ist der Landkreis Diepholz mit vielfältigen Herausforderungen des demographischen Wandels konfrontiert. Dazu zählen beispielsweise eine geringe Geburtenrate und Bevölkerungsrückgang mit hohem Anteil an älteren Menschen, aber auch eine hohe Bildungsabwanderung. ${ }^{3}$ Im Zeitraum von 2010 bis 2014 profitierten die Städte und Gemeinden des Landkreises von (Aus-)Bildungszuwanderung, wenn sie in der Nähe von Arbeitsplatz- und Ausbildungszentren liegen. Dazu zählen etwa die Gemeinden Stuhr und Weyhe, aber auch die Kreisstadt Diepholz mit einer privaten Hochschule und die Samtgemeinde Wagenfeld (Landkreis Diepholz 2016: 15). Auch machen die Samtgemeinde Rehden mit regionaler und überregionaler Bedeutung für Erdgas und die Samtgemeinde Altes Amt Lemförde mit den großen Arbeitgebern wie BASF Polyurethanes $\mathrm{GmbH}$ und ZF Lemförder Fahrwerktechnik die Bedeutung der Kommunen für den Arbeitsmarkt deutlich. Aufgrund der guten Arbeitsmarktsituation im Landkreis Diepholz ist demnach davon auszugehen, dass der Landkreis wochentags durch temporäre Anwesenheiten von multilokal lebenden "Incomings“ (Lange 2018: 176), also solchen, die zeitweise berufsbedingt in den Landkreis kommen, betroffen ist.

Eine Haushaltsbefragung von Bewohnerinnen/ Bewohnern des südlichen Teils des Landkreises im Rahmen des Forschungsprojektes "TempALand“ ergab, dass es eine nicht unwesentliche Anzahl multilokal lebender Menschen im Landkreis Diepholz gibt (Greinke/ Lange/Othengrafen 2017: 18 ff.; Greinke/Lange/Othengrafen 2018: 45). Im Rahmen des Projektes wurden 362 multilokal Lebende schriftlich befragt. Zudem wurden 65 qualitative Interviews geführt. Aufgrund von „verdeckter Multilokalität“ oder „Kryptomultilokalität“ (Weichhart/ Rumpolt 2015: 17 f.) ist davon auszugehen, dass die Anzahl der Multilokalen noch größer ist.

3 https://www.wegweiser-kommune.de (26.04.2019). 


\subsection{Das Forschungsdesign}

Das Untersuchungsgebiet wurde mittels Ortsbegehungen und Dokumentationen charakterisiert. Danach wurden eine Online-Befragung und problemzentrierte leitfadengestützte Interviews (nach Meuser/Nagel 2002; Helfferich 2011; Mayer 2013) unter Einbezug von Elementen der reflexiven Fotografie (nach Dirksmeier 2009: 164) mit multilokal lebenden Beschäftigten durchgeführt, ergänzt durch problemzentrierte Interviews mit Personen aus der Führungsebene der Unternehmen (z. B. Abteilungsleiter oder Personalabteilung) sowie Personen aus Planung, Politik und Verwaltung, zumeist aus der Wirtschaftsförderung und Bürgermeistern. Diese Akteure sind in Bezug auf Multilokalität im Untersuchungsgebiet relevant, weil sie entweder selbst multilokal leben, Multilokale im Unternehmen beschäftigen oder von mehrörtigen Lebensweisen berührt sind. Im Rahmen des Dissertationsvorhabens wurden qualitative Interviews geführt mit 14 multilokal lebenden Personen und acht politisch-planerischen Akteuren. Sie wurden jeweils zu ihrer mehrörtigen Lebensweise als auch zu betrieblichen und kommunalen Strukturen befragt. Im vorliegenden Beitrag werden ein Zwischenfazit gezogen und erste Tendenzen aufgezeigt.

Die multilokal lebenden interviewten Personen wurden mittels einer Online-Befragung oder über Poster und Flyer, die postalisch an die Unternehmen gesendet und beispielsweise in Eingangsbereichen, Kantinen und stark frequentierten Orten in den Unternehmen ausgehängt und ausgelegt wurden, angeworben. Weitere interviewte Personen wurden mittels Schneeballverfahren gewonnen. Die multilokalen Interviewten haben sich freiwillig aufgrund des Aufrufs durch die Poster und Flyer gemeldet. Als politisch-planerische Akteure wurden die Bürgermeister der Untersuchungskommunen ausgewählt.

Vor dem Gespräch wurden die multilokal lebenden Befragten in einem telefonischen Initialinterview gebeten, zu ihrem Alltagsleben an den verschiedenen Orten jeweils drei Fotoaufnahmen anzufertigen und reflektierende Notizen zu den Fotos zu machen (Dirksmeier 2007: 87). In den anschließenden Interviews wurden die Fotos als eine Art "starting mechanism“ oder Erzählimpuls (Dirksmeier 2007: 88; Brake 2009: 379) genutzt. Die Methode der reflexiven Fotografie der qualitativen Sozialraumforschung stammt aus den Cross Cultural Studies und kombiniert Interview- und Fotografieverfahren zu einer kohärenten Methodik (Dirksmeier 2007: 87; Brake 2009: 378), in der die Rollen der interviewten Personen und der wissenschaftlich Beobach- tenden vertauscht werden (Dirksmeier 2009: 168; Brake 2009: 384 f.). Dadurch treten die Interviewten als Expertinnen/Experten für ihre Fotografien und ihre Lebenswelten auf, berichten von ihren Intentionen und Interpretationen und wählen eigenständig und subjektiv Motive aus (Krisch 2002: 133). Sie werden zum bildlichen Nachdenken angeregt und machen reflektierte Angaben über ihre Aufnahmen (Dirksmeier 2009: 168).

Im weiteren Gesprächsverlauf wurden Anforderungen an die Wohnung und das Wohnumfeld wie Ausstattung, Lage und Größe der Wohnungen sowie Merkmale der Lebensweise wie Distanzen und Nutzungsdauer thematisiert. Außerdem wurden die mehrörtigen Lebensweisen vor Ort erläutert (z. B. Arbeits- und Alltagsleben, Infrastrukturnutzungen, bürgerschaftliches Engagement). Des Weiteren wurde über Multilokalität in den Unternehmen gesprochen (z. B. Verbreitung, Wahrnehmung, zukünftige Entwicklungen).

Themen in den Interviews mit den Bürgermeistern waren Informationen zu den Kommunen (z. B. aktuelle Handlungsfelder, Infrastrukturausstattung, Wohnungsmarktsituation, Ortsgemeinschaften) sowie Multilokalität in den Kommunen (z. B. Motive, Vorkommen, Wahrnehmung, Chancen und Risiken) sowie Handlungsspielräume. Ausgewertet wurden alle Interviews mittels qualitativer computergestützter Inhaltsanalyse (Software MAXQDA) (nach Meuser/Nagel 2002; Mayring 2010).

\section{Berufsbedingte Multilokalität im Landkreis Diepholz}

Nachfolgend werden die (Zwischen-)Ergebnisse aus den Interviews mit den multilokal lebenden Beschäftigten sowie den Bürgermeistern vorgestellt. Insgesamt wurden 12 männliche und zwei weibliche berufsbedingt multilokal lebende Personen sowie acht männliche Bürgermeister interviewt. Das Durchschnittsalter der interviewten Multilokalen liegt bei rund 50 Jahren. Neun interviewte Personen haben jeweils ein, zwei oder drei Kinder, von denen nur vereinzelt Kinder im Teenageralter oder der Phase des Berufseinstieges am Nebenort sporadisch (z. B. in den Ferien) anwesend sind. Beruflich sind die Interviewten in Industrieunternehmen (Automobilzulieferer, Getränke, Gastronomie, Rohstoff) tätig, in denen 12 Personen leitende Tätigkeiten und drei ausführende (gehobene) Tätigkeiten ausüben. Die Wohnorte der interviewten Multilokalen liegen überwiegend in Deutschland (vgl. Abbildung 1). Sie benennen ihren Wohnort im Landkreis Diepholz als Nebenort, der 


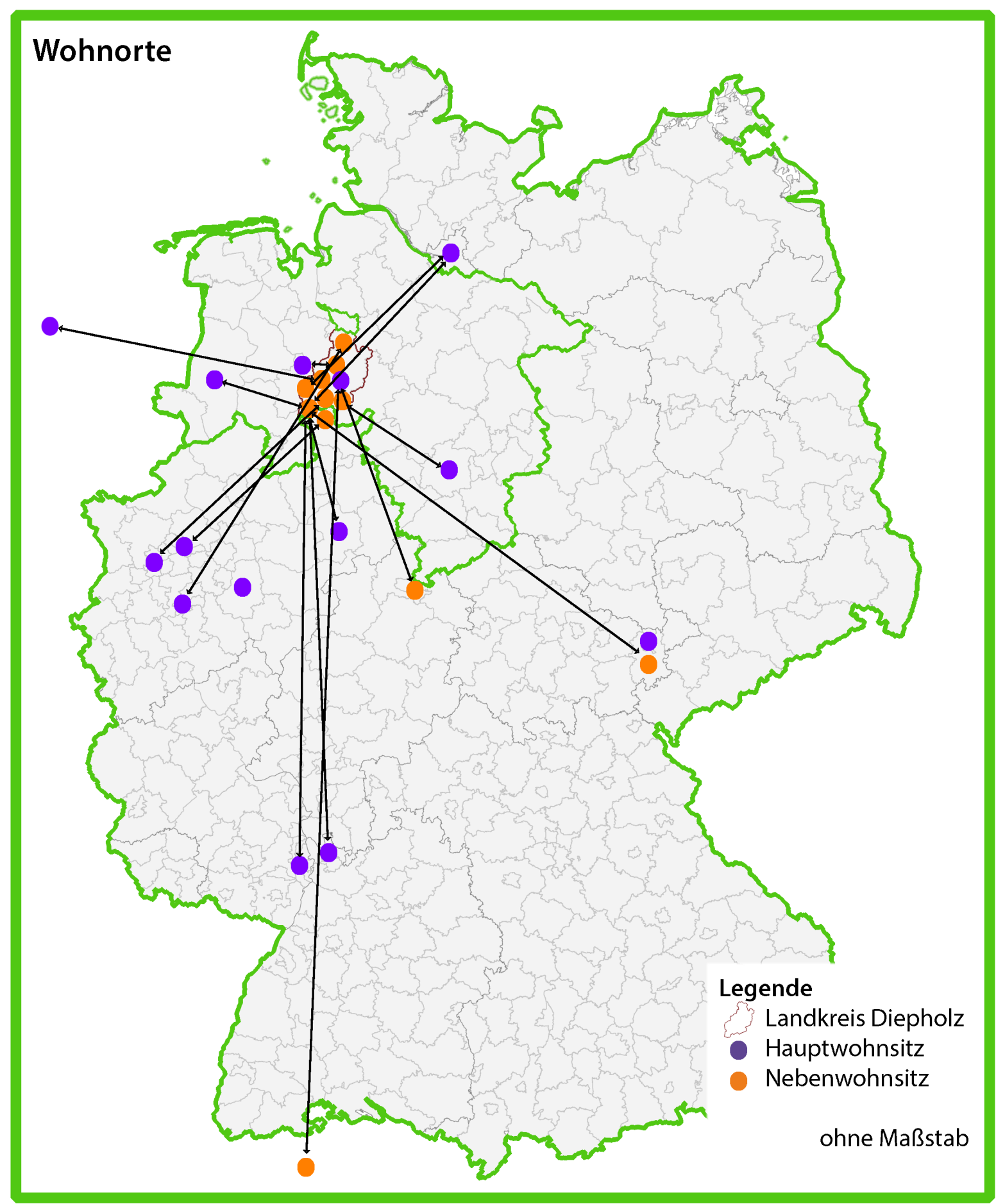

Abbildung 1: Wohnorte der befragten Personen

Kartengrundlage verändert nach GeoBasis-DE/BKG 2014 
zumeist Arbeitsort ist. Lediglich ein freizeitbedingter Nebenort und zwei beruflich bedingte Nebenorte befinden sich außerhalb des Landkreises Diepholz. Ein Ausgangsort, also der von den Multilokalen ersterschlossene Ort, befindet sich in den Niederlanden. Anders als andere Untersuchungen zeigen (z. B. Peer 2013), liegen in diesem Beitrag die Ausgangsorte der Interviewten entweder in Klein-, Mittel- oder Großstädten und die Nebenorte in einer Landgemeinde oder (kleineren) Kleinstadt. ${ }^{4}$ Dementsprechend sind die Wohnorte in Bezug auf Größe und Zentralität divergent. Die Arbeitsorte liegen in eher ländlich geprägten Kommunen. 12 der befragten Personen mieten am Nebenort eine Zweitwohnung und zwei übernachten regelmäßig im zumeist gleichen Hotel. Fünf Interviewte leben weniger als vier Jahre multilokal im Landkreis Diepholz, sechs Multilokale leben fünf bis neun Jahre und drei interviewte Personen leben mehr als zehn Jahre mehrörtig. Zwischen den Orten wechseln die Multilokalen zumeist an den Wochenenden, sodass sie in der Regel innerhalb der Woche im Landkreis Diepholz anwesend sind und an den Wochenenden an ihren anderen Ort zurückkehren.

\subsection{Temporär genutzte Räume aus der Sicht der multilokal Lebenden}

Die Ansprüche und Bedürfnisse multilokal lebender Personen in ländlichen Räumen sind sehr divers (vgl. Lange 2018). Daher stellen sich die Fragen: Welche Beweggründe und Umstände sorgen dafür, dass Menschen an mehreren Orten leben (müssen)? Wie gestalten mehrörtig lebende Personen ihr Alltags- und Berufsleben sowie inr soziales Leben, das heißt auch, wie stellen sie ihre temporär genutzten Räume immer wieder aufs Neue her? Und welche betrieblichen Rahmenbedingungen und Strukturen beeinflussen diese Lebensweisen?

\subsubsection{Beweggründe und betriebliche Rahmenbedingungen}

Die Beweggründe der Multilokalen sind alle zunächst berufsbedingt, wobei es darüber hinaus deutliche individuelle Unterschiede gibt. Während bei acht Personen diese Lebensweise primär privat motiviert ist, etwa weil

4 Gemäß Stadt- und Gemeindetypen des Bundesinstituts für Bau-, Stadt- und Raumforschung; vgl. https://www.bbsr.bund.de/BBSR/ DE/Raumbeobachtung/Raumabgrenzungen/StadtGemeindetyp/ StadtGemeindetyp_node.html (26.04.2019). sie regelmäßig bei ihrem Partner oder ihrer Partnerin wohnen und dadurch zu ihrem Arbeitsort weitere Entfernungen zurücklegen müssen, gibt es sieben Personen, die die Lebensweise aus primär beruflichen Anlässen gewählt haben, z. B. weil sie von ihren Arbeitgebern delegiert worden sind, um an einem anderen Betriebsstandort z. B. Projekte zu initiieren oder zu leiten. Wollen die Beschäftigten andere, oft höhere Positionen in den Unternehmen besetzen, so sind sie in einer gewissen Form dazu gezwungen, Erfahrungen an anderen Betriebsstandorten zu sammeln und dadurch phasenweise multilokal zu leben.

Solche Fälle von Multilokalen finden sich auch im Landkreis Diepholz: Aufgrund der guten Arbeitsmarktsituation mit attraktiven Unternehmen entscheiden sich einige Beschäftigte, dort einen weiteren Wohnsitz anzunehmen. Zusätzlich sind vier der interviewten Personen aufgrund ihrer leitenden Tätigkeit auf Dienstreisen innerhalb Deutschlands und weltweit unterwegs, wodurch sich ihre ohnehin sehr mobile Lebensweise noch verstärkt. Insgesamt sind die Arbeitsweisen in den Betrieben der interviewten Multilokalen durch zeitweise Projektarbeiten und Delegationen sowie zusätzliche Dienstreisen geprägt und somit fördern die Unternehmen multilokale Lebensweisen.

Hinter der mehrörtigen Lebensweise stehen zwar einerseits häufig Zwänge, andererseits wird den Beschäftigten diese Lebensweise oft auch erst durch betriebliche Rahmenbedingungen und Strukturen ermöglicht. Denn durch flexible Arbeitszeiten, etwa in Form von Gleitzeit, die bei vielen Betrieben im Landkreis Diepholz gewährt wird, können die Beschäftigten ihre Arbeitsstunden zu einem gewissen Grad frei verteilen. Zudem wird diese Flexibilität durch Unterstützungsleistungen, wie beispielsweise eine Bahncard, die ein Unternehmen im Landkreis Diepholz Multilokalen zur Verfügung stellt, positiv beeinflusst. Des Weiteren kann der Einsatz digitaler Informations- und Kommunikationstechnologien zukünftig die Standortunabhängigkeit erhöhen. Durch Heimarbeitsregelungen und Telefon- oder Videokonferenzen wird die Kommunikation möglicherweise erleichtert und weniger physische Mobilität, z. B.in Form von Dienstreisen, notwendig. Dies kann vor allem für ländliche Räume sowohl Risiko als auch Chance sein: Einerseits sind Multilokale, die in diese Räume eigentlich berufsbedingt kommen würden, länger nicht vor Ort und andererseits können Multilokale, die berufsbedingt innerhalb der Woche ländliche Räume verlassen würden, länger vor Ort bleiben und damit auch die dortigen Infrastrukturen und Dienstleistungen nutzen. 


\subsubsection{Raum-zeitliche Gestaltung von temporärer Nähe}

In der Anfangsphase der Anwesenheit im Landkreis Diepholz, z. B. während der Probezeit, wohnen die interviewten Beschäftigten zunächst in Hotels, Boardinghäusern oder zur Untermiete, oft im näheren Umfeld der Betriebe. Danach suchen sich die meisten eine andere Unterkunft, mit Ausnahme von zwei Personen, die dauerhaft im selben Hotel im Landkreis Diepholz leben. Dabei bevorzugen sie Wohnungen, die in unmittelbarer Nähe zu ihrer Arbeitsstelle und zu Einkaufsmöglichkeiten liegen: „Ich habe natürlich schon etwas gesucht, was direkt neben der Firma ist. Weil ich jetzt hier nicht auch noch viel fahren kann" (48-jähriger männlicher Multilokaler mit berufsbedingtem Nebenort im Landkreis Diepholz). Der (phasenweise) Bezug der Unterkünfte kann möglicherweise besonders für ländliche Räume vorteilhaft sein, weil somit zumindest (phasenweise) Wohnraum belegt wird, der ansonsten gegebenenfalls leer steht.

Die favorisierten Unterkünfte sind zumeist eher klein und werden häufig gerne möbliert angemietet. Die von den Beschäftigten als Nebenort klassifizierte Unterkunft wird zudem oft funktional, schlicht und einfach eingerichtet, sodass lediglich eine spartanische Ausstattung mit den nötigsten Möbeln und Haushaltsgeräten vorhanden ist und sie für die Zeiten der Anwesenheit ihre persönlichen Gegenstände, wie beispielsweise Kleidung und vereinzelt auch Lebensmittel, mitbringen. Dieser Aspekt kann für ländliche Räume nachteilig sein, da die Multilokalen durch die Mitnahme der Lebensmittel nicht vor Ort einkaufen und dadurch der Einzelhandel in ländlichen Räumen keine (zusätzlichen) Einnahmen gewinnt.

$\mathrm{Da}$ die mehrörtig lebenden Beschäftigten am Nebenort in unregelmäßigen Rhythmen anwesend sind, unterscheiden sich auch die Nutzungen der Infrastrukturen und Dienstleistungen stark. Zumeist nutzen sie am Nebenort lediglich die Einkaufsmöglichkeiten für Lebensmittel und vereinzelt den Bahnhof für die Anund Abreise. Dienstleistungen werden am Nebenort eher selten in Anspruch genommen. Auch Arztbesuche machen die mehrörtig Lebenden zumeist am Ausgangsort und nehmen diese Leistungen nur in Notfällen im Landkreis Diepholz in Anspruch. Allerdings gibt es auch vereinzelt Multilokale, die das Angebot am Nebenort im Landkreis Diepholz schätzen gelernt haben und deshalb einige Dienstleistungen, wie beispielsweise Kosmetikbehandlungen, aufgrund des guten Preis-LeistungsVerhältnisses ausschließlich dort in Anspruch nehmen. Attraktive Dienstleistungen können demnach für länd- liche Räume vorteilhaft sein, weil sie Multilokale (und Zuziehende) anziehen können. Obwohl die Gemeinden des Landkreises Diepholz vereinzelt durch Bahnhöfe erschlossen sind, fehlt es in etlichen an einem geeigneten öffentlichen Verkehrsangebot. Aus diesem Grund reisen nahezu alle Befragten mit dem privaten oder betrieblichen Personenkraftwagen an. Lediglich eine Person reist ausschließlich mit der Bahn und benutzt vor Ort ein Fahrrad zur Fortbewegung.

Raum-zeitlich betrachtet reisen die Multilokalen oft am Montagmorgen oder sogar erst gegen Mittag an und bereits am Donnerstagabend oder spätestens Freitagmittag wieder ab. Teilweise unterscheidet sich aber der Wochenrhythmus in dieser Stichprobe stark (vgl. Leubert 2013: 98) und wird oft durch soziale oder betriebliche Rahmenbedingungen (wie Kinderbetreuung, Pflege von Angehörigen oder Dienstreisen) beeinflusst. Die Interviewten versuchen zwar, einen festen Reiserhythmus aufzubauen, allerdings gelingt dies oft nicht. Gründe hierfür sind beispielsweise die Krankheit eines Familienangehörigen, aber auch berufliche Restriktionen wie Dienstreisen: „Ich wollte eigentlich immer Dienstag, Mittwoch, Donnerstag hier sein, aber ehrlich gesagt ist das meistens nicht der Fall, weil ich dann doch irgendwo anders bin. Aber es war eigentlich das Ziel [lacht]“ (48-jähriger männlicher Multilokaler mit berufsbedingtem Nebenort im Landkreis Diepholz).

\subsubsection{Zusammenleben bei temporärer Nähe}

Innerhalb der Woche sind die Multilokalen in diesem Sample oft einer hohen Arbeitsbelastung ausgesetzt, weil sie zumeist leitende Tätigkeiten haben (vgl. Jurczyk/Schier/Szymenderski et al. 2009; Leubert 2013) und sie ihre Arbeitszeit auf wenige Tage in der Woche verteilen (vgl. Leubert 2013: 103). Zudem konzentriert sich deshalb das Sozialleben derjenigen untersuchten Multilokalen, die Familie haben, nahezu ausschließlich auf die Wochenenden (vgl. Leubert 2013: 150). In der Woche der Anwesenheit im Landkreis Diepholz planen die Beschäftigten gelegentlich Unternehmungen mit ihren Kolleginnen/Kollegen. Diese finden zumeist in den Abendstunden statt und beschränken sich auf Restaurantbesuche oder Betriebssportangebote wie Fahrradgruppen. Somit stellen die mehrörtig Lebenden für ländliche Räume durchaus ein Potenzial dar, weil sie in den Zeiten der Anwesenheit die lokale Wirtschaft nutzen (Restaurantbesuche und anderes), allerdings sind sie aufgrund der hohen Arbeitsbelastung und damit geringeren Freizeit eine eher kleine Zielgruppe. Über die 
sozialen Interaktionen finden sich die Multilokalen gut in die Kollegenschaft ein. Als Freundschaft bezeichnen die Personen die Kontakte jedoch nicht, wodurch deutlich wird, dass sie im Bereich des sozialen Lebens eine klare Trennung zwischen den Orten vornehmen. Oft passiert dies bewusst, denn bedauert wird diese Tatsache eher selten. In Bezug auf die Funktionen der Wohnungen ist dieses Auseinanderhalten weniger deutlich: Obwohl die meisten Wohnungen der Beschäftigten am Nebenort eher funktional eingerichtet sind, werden sie an den Wochenenden und im Urlaub von der Familie oder den Partnerinnen/Partnern mitbenutzt: „Wir haben auch Freunde hier im Norden. Also so Bremen und Hamburg, und dann verbringen wir auch schon mal Wochenenden hier. Und dann kommt sie [seine Frau] halt hier vorbei und dann ist es natürlich praktisch, wenn man hier eine Wohnung hat" (53-jähriger männlicher Multilokaler mit berufsbedingtem Nebenort im Landkreis Diepholz). In der Freizeit kommen folglich des Öfteren Familienangehörige in den Landkreis Diepholz und verbringen dort Wochenendtrips oder ihre Ferien. Zwei mehrörtig lebende Personen haben sich aus diesem Grund bereits eine größere Unterkunft gesucht, um Gäste zu empfangen oder die Freizeit dort zu verbringen. Darin liegt vor allem für ländliche Räume eine Chance, das Leben für die phasenweise Anwesenden angenehm und attraktiv zu gestalten und sie dadurch gegebenenfalls zum Zuzug zu bewegen und damit an Bevölkerung zu gewinnen.

\subsection{Die Bedeutung berufsbedingter Multilokalität für das Zusammenleben sowie betriebliche und regionale Entwicklungen in ländlichen Räumen}

Die subjektorientierte Erforschung multilokaler Lebensweisen im Landkreis Diepholz zeigt eine Bandbreite an Beweggründen, Ausprägungsformen und Lebensführungen, die mit diesem mehrörtigen Dasein einhergehen. Ein Wechsel der Perspektive führt nun zu den Fragen: Welche Bedeutung kann diese berufsbedingte Multilokalität für betriebliche und regionale Entwicklungen haben? Auf welche Herausforderungen müssen sich Betriebe und Akteure der Regionalentwicklung voraussichtlich einstellen? Und welche Chancen und Risiken ergeben sich durch die mehrörtig lebenden Beschäftigten aus der Sicht der Betriebe und der Regionalentwicklung?

\subsubsection{Soziale Interaktionen}

Einige mehrörtig lebende Beschäftigte pflegen diese Lebensweise im Landkreis Diepholz bereits über lange Zeiträume (10 bis 15 Jahre), wohingegen andere erst kürzer an mehreren Orten leben (1 bis 5 Jahre). Zumeist haben die Beschäftigten jedoch auch zuvor mehrörtig gelebt und sich somit an diese Lebensweise gewöhnt oder mindestens angepasst. Auch die Familie und Angehörige sowie Freunde und Bekannte haben sich oftmals bereits auf die Lebensweise eingestellt und wissen, dass die multilokal Lebenden nur an den Wochenenden an ihrem Ausgangsort anwesend sind. Die temporäre Abwesenheit führt jedoch dazu, dass sie zum Teil aus dem Sozialleben ausgeschlossen werden. Beispielsweise äußern sie in den Interviews, dass sie zu Geburtstagen oder anderen Feierlichkeiten gar nicht erst eingeladen werden, weil sie ohnehin nicht da wären.

Die Interviewten können sich diese Lebensweise nicht als eine dauerhafte Lösung vorstellen und wollen später (wieder) beruflich monolokal leben. Dann wollen sie, falls es sich ergibt, lediglich freizeitbedingt an mehreren Orten leben. Ein Ort im Landkreis Diepholz kommt dafür jedoch zumeist nicht in Frage. Sie definieren in der Regel ihren Ausgangsort als Heimatort und identifizieren sich auch mit der dortigen Gemeinschaft: „Das [Bild] mit der Feuerschale war das Osterfeuer bei uns. Da waren alle Freunde und die Familie da. Das ist mir wichtig und deswegen wäre für mich die Entscheidung auch immer klar, dass ich mein Leben nicht hier [im Landkreis Diepholz] verbringen möchte. Sondern mein Hauptstandort, wo ich immer wohne, eigentlich bei mir zu Hause ist. Da wo ich auch aufgewachsen bin. Mein Zuhause ist in [am Ausgangsort außerhalb des Landkreises Diepholz]" (32-jährige weibliche Multilokale mit berufsbedingtem Nebenort im Landkreis Diepholz). Hier gilt es für ländliche Kommunen, attraktiv zu sein und die Multilokalen zum Zuzug zu gewinnen, um beispielsweise im Bereich des Einzelhandels oder auch durch kommunale Finanzzuweisungen zu profitieren.

Dennoch bemerken die Befragten, dass der Arbeitsmarkt es derzeit und zukünftig vermutlich nicht zulassen wird, dass sie nahe dem Ausgangsort eine Arbeitsstelle finden. Aus diesem Grund befürchten sie, noch lange an mehreren Orten leben zu müssen und damit weiterhin nur eingeschränkt am Sozialleben am Ausgangsort teilhaben zu können. Es kann vermutet werden, dass zusätzliche Wechsel der Multilokalen aufgrund von zeitlich begrenzter Projektarbeit, Dienstreisen oder Delegationen diese Situation zunehmend verschärfen. Denn dann fehlen neben den Kontakten am Ausgangsort auch 
zusätzlich noch Interaktionen an den Nebenorten, weil diese aufgrund kurzer Verweildauer und häufig wechselnder An- und Abwesenheiten gar nicht erst entstehen oder nur schwer aufrechterhalten werden können. Auf der anderen Seite jedoch, so zeigen die Analysen, kann sich dieser Aspekt auch positiv auf das soziale Leben der Multilokalen auswirken, denn durch die vielen temporären Aufenthalte können sie beispielsweise vermehrt neue Bekanntschaften machen und ihr Netzwerk ausbauen. Auf dieses können sie dann zurückgreifen.

Trotz starker zeitlicher Restriktionen sind einige Beschäftigte schon seit Längerem an ihrem Ausgangsort engagiert und nehmen für das Engagement sogar häufigere Reisen in Kauf. Wenn beispielsweise ein Fußballspiel innerhalb der Woche am Ausgangsort angesetzt ist, so reisen die Interviewten teilweise für wenige Stunden zurück, um als Spielende aktiv daran teilzunehmen, und kehren nach dem Spiel wieder zurück an ihren Nebenort. Eine Rückkehr ist oft auch aus familiären Gründen phasenweise und spontan nötig. Beispielsweise pflegen einzelne Multilokale ihre Angehörige. Am Nebenort hingegen beschränkt sich ihr Engagement nahezu ausschließlich auf Tätigkeiten im Zusammenhang mit der Arbeitsstelle, wie etwa die Leitung einer Firmensportgruppe. Oft bleibt jedoch kaum Zeit für ein Engagement am Nebenort, sodass hier keine Interaktionen zwischen der Kollegenschaft oder der übrigen örtlichen Bevölkerung stattfinden. Somit bleiben intensive Interaktionen am Nebenort aus und am Ausgangsort können Kontakte nur erschwert aufrechterhalten werden. Diese Nachteile nehmen Multilokale oftmals nur in Kauf, weil sie finanziell durch eine bessere Beschäftigung profitieren.

\subsubsection{Betriebliche Strukturen}

Die Multilokalen erklären, dass die Betriebe im Landkreis Diepholz zumeist mehrere Standorte haben und zusätzlich noch vermehrt Projektarbeiten, Dienstreisen und Delegationen veranlassen. Durch die Delegationen und befristeten Arbeitsverhältnisse können die Betriebe als eine Art Treibkraft für multilokale Lebensweisen identifiziert werden, weil sie Beschäftigte phasenweise an andere Standorte der Unternehmen entsenden. Vermuten lässt sich, dass die Multilokalen aufgrund attraktiver Arbeitsbedingungen oder lohnenswerter Vergütung durchaus gewillt sind, befristete Delegationen oder Projektarbeiten an zusätzlichen anderen Orten anzunehmen und demnach ihrem Unternehmen ,treu bleiben anstatt ihr Beschäftigungsverhältnis aufzugeben. Durch diese Delegationen treiben jedoch die Unternehmen die
Mehrörtigkeit voran, weil die Multilokalen sich häufig ein Lebensumfeld an einem Ausgangsort aufgebaut haben, welches sie für befristete Delegationen oder Projektarbeiten nicht aufgeben wollen. Eine multilokale Lebensweise kann dann eine Möglichkeit sein, mit den betrieblichen Entsendungen umzugehen. Betriebliche Entsendungen können demnach voraussichtlich eine Zunahme mehrörtiger Lebensweisen implizieren. Zusätzliche Dienstreisen (an wiederkehrende Orte) können diese Mehrörtigkeit verstärken. Im ersten Schritt steht für die Beschäftigten die Wahl der beruflichen Tätigkeit im Vordergrund und nicht etwa eine Standortwahl von Arbeits- und Wohnort. Dies kann eine Chance für die Betriebe bedeuten, denn wenn sie (beruflich) attraktiv genug für die multilokalen Beschäftigten sind, bekommen sie immerhin phasenweise Zulauf und müssen sich unter Umständen weniger Sorgen um Nachwuchskräfte machen. Denkbar ist auch, dass die zunächst mehrörtig lebenden Beschäftigten ihre Lebensweise nach einer Zeit aufgeben und vollständig an den Nebenort ziehen. Die Bürgermeister einiger Gemeinden haben dies bereits beobachtet. Zumeist erfolgte ein Komplettumzug zwar erst nach Aufgabe der beruflichen Tätigkeit, um den Ruhestand in ländlich attraktiven Gebieten zu verbringen, aber es besteht vermutlich durchaus Potenzial, dass dies bereits früher im Lebenslauf geschehen kann. Dadurch könnten vor allem ländliche Räume mit attraktivem landschaftlichem oder kulturellem Angebot von Zuzug profitieren.

Es lässt sich außerdem annehmen, dass sich die Betriebe auch der Herausforderung stellen müssen, dass die Beschäftigten sich aufgrund der mehrörtigen Lebensweise (noch) weniger an die Betriebe binden beziehungsweise gebunden werden. Sind die Multilokalen beispielsweise über Projekte beschäftigt oder sind die Auftragslagen in den Unternehmen ungewiss, so binden die Betriebe die Beschäftigten über befristete Arbeitsverhältnisse nicht langfristig. Anders herum binden sich die Multilokalen durch befristete Beschäftigungen weniger stark und halten sich so offen, ihre mehrörtige Lebensweise schneller wieder aufzugeben. Die Befristungen können somit dazu führen, dass die Beschäftigten die berufliche Tätigkeit aufgeben beziehungsweise eine andere Tätigkeit aufnehmen, und zwar noch rascher als es monolokal wohnende, befristet Beschäftigte tun würden. Daraus entsteht ein hohes Risiko für die Betriebe, die bei personellen Wechseln zeitnah für eine Nachbesetzung sorgen müssen und bei denen sogar ganze Projekte an Rekrutierungsschwierigkeiten scheitern können. Für Betriebe in ländlichen Regionen, die ohnehin oft mit ,Nachwuchssorgen' konfrontiert sind, stellt sich diese Herausforderung zugespitzt dar. 
Aus den Interviews mit den Bürgermeistern wird deutlich, dass es für die Unternehmen im ländlich geprägten Landkreis Diepholz zunehmend schwieriger ist, geeignete Fachkräfte und junge Menschen zu gewinnen. Vor allem letztere verlassen den Landkreis, um in einer größeren Stadt zu studieren oder eine Ausbildung zu absolvieren. Für ländliche Räume ist die Abwanderung (hochqualifizierter) Arbeitskräfte oft mit negativen Folgen verbunden (Peer 2013: 2). Multilokale Beschäftigte können hier eine Chance sein, den Fachkräftemangel abzumildern (Greinke/Lange/Othengrafen 2018: 46). Können die Betriebe beispielsweise Beschäftigte zumindest phasenweise gewinnen, werden diese gegebenenfalls sogar zu Zuziehenden oder sie decken immerhin für kürzere Zeiträume Personalengpässe ab. Die Betriebe müssen dann aber attraktiv für ihre Beschäftigten sein, was manche etwa über Sonderleistungen bereits umsetzen: Einige stellen beispielsweise Dienstwagen oder Diensthandy zur Verfügung, andere ermöglichen Betriebssport und wieder andere haben individuelle Unterstützungsleistungen, die sie zum Teil direkt auf die einzelnen Beschäftigten zuschneiden. Für die Multilokalen mit Kindern kann das Freihalten von Kinderbetreuungsplätzen oder Heimarbeitsregelungen attraktiv sein, da innen dies die Betreuung der Kinder während der Arbeitszeit erleichtert. Auch wenn die Stichprobe keine Fälle hergibt, in denen zu betreuende Kinder in den Landkreis Diepholz mitkommen, haben die Interviews mit den Bürgermeistern ergeben, dass es bereits Multilokale gab, die freigehaltene Kinderbetreuungsplätze in Anspruch genommen haben. Die multilokalen Lebensweisen können die Unternehmen also auch anregen, neue betriebliche Angebote zu schaffen oder für eine bessere Auslastung bisheriger betrieblicher oder kommunaler Angebote zu sorgen.

\subsubsection{Regionale Entwicklungen}

In Bezug auf die regionale Entwicklung lässt sich weiters vermuten, dass es eine Chance für die Gemeinden des Landkreises Diepholz sein kann, wenn multilokal Lebende ihr Arbeits- und Privatleben nicht räumlich trennen. Dadurch, dass die Interviewten teilweise ihre Unterkunft gemeinsam mit inrer Familie oder Freunden nutzen, die zu Besuch kommen, können die Kommunen profitieren. Sowohl an den Wochenenden als auch in den Schulferien sind Familienangehörige im Landkreis Diepholz anwesend und verbringen ihre Freizeit in Naherholungsgebieten, beispielsweise am Dümmer See, oder beleben den Einzelhandel durch ihre Einkäufe.
Dadurch können Multilokale für Kaufkrafttransfer sorgen (Peer 2013: 2; ARL 2016: 15).

Zudem sind die mehrörtig lebenden Beschäftigten trotz einer funktionalen Ausstattung an einer eher hochwertigen und attraktiven Gestaltung ihrer Wohnorte interessiert. Dabei unterscheiden sich die Definitionen einer attraktiven Wohnung: Einige bevorzugen eine schlichte und einfache Ausstattung der Wohnung, wohingegen andere beispielsweise Wert auf historische Bausubstanzen legen. Nichtsdestotrotz ist für Multilokale der Landkreis Diepholz so attraktiv, dass zwei Befragte sogar Eigentum am Nebenort besitzen. Dies ist zum einen durch die preisgünstigen Anschaffungskosten, zum anderen aber auch durch die Attraktivität der Region mit hohem Freizeitwert begründet. Die Multilokalen sind folglich durchaus bereit, sich im Landkreis Diepholz 'fester' niederzulassen, wodurch die Kommunen durch (phasenweisen) Zuzug profitieren.

Allerdings ist es den mehrörtig lebenden Beschäftigten oft nicht leichtgefallen, passenden Wohnraum zu finden (vgl. Greinke/Lange/Othengrafen 2018). Aufgrund der attraktiven Lage mit touristischen Zielen im Landkreis Diepholz überlegen einige Multilokale, ihre Wohnungen während ihrer Abwesenheit zur Zwischenmiete anzubieten. Dies birgt ein großes Risiko für die ansässigen Beherbergungsbetriebe, weil dadurch Konkurrenzen entstehen können. Erste Ansätze in Bezug auf die Wohnungsmarktentwicklungen im Landkreis Diepholz werden darin deutlich, dass bereits zwei Boardinghäuser errichtet wurden, die auf die Bedürfnisse der phasenweise Anwesenden abgestimmt sind. Boardinghäuser bieten flexible Mietdauern und eingerichteten Wohnraum, der auch für wenige Wochen oder Monate vermietet wird.

In Bezug auf die Freizeitinfrastrukturen können multilokale Lebensweisen voraussichtlich belebend wirken. Einige Multilokale schaffen sich im Landkreis Diepholz explizit kleine Freiräume nach Dienstschluss an den oft recht vollen Arbeitstagen, um inrem Hobby oder einer Freizeitbeschäftigung nachzugehen, z. B. Fahrradfahrten oder Restaurantbesuche mit Kolleginnen/Kollegen. Diese Freizeitgestaltung üben sie normalerweise nicht oder nur sehr selten am Ausgangsort aus. Die Multilokalen können also allemal ein Potenzial für Vereine und Institutionen sein, weil sie durchaus gewillt sind, ihre knappe Zeit für Freizeitbeschäftigungen am Nebenort zu investieren. Hierzu bedarf es aber auch eines Umdenkens hin zu flexiblen Angeboten hinsichtlich Vereinsstrukturen, die mancherorts noch sehr starr sind und die Mitwirkung temporär Anwesender kaum ermöglichen. 


\subsection{Handlungsfelder für betriebliche und regionale Entwicklungen}

Multilokale Lebensführungen sind in soziale, betriebliche und regionale Zusammenhänge eingebunden und bergen für diese sowohl Chancen als auch Risiken. Hieraus lassen sich für alle drei Ebenen - das Zusammenleben, die Betriebe und die Regionen - exemplarisch erste Handlungsfelder aufzeigen.

\subsubsection{Gestaltung des Zusammenlebens}

Da einige Multilokale ihre Lebensweise bereits seit einigen Jahren führen, nehmen sie nicht mehr so aktiv am Sozialleben am Ausgangsort teil. Hier ist es von Vorteil, wenn am Nebenort für Interaktionen geeignete (physische) Räume zur Verfügung gestellt werden, in denen sich multilokal Lebende mit ihrer Kollegenschaft (regelmäßig) austauschen können. Die Interviews mit den Multilokalen haben gezeigt, dass sie am Nebenort ein soziales Netzwerk innerhalb der Kollegenschaft aufbauen. Für den Austausch untereinander können zunächst der Bedarf geprüft und danach geeignete Zeiten und Räume auch außerhalb der Normalarbeitszeit - vielleicht über ein betriebliches Freizeitangebot geschaffen werden. Hier besteht die Möglichkeit, an die bestehenden Angebote der Unternehmen im Landkreis Diepholz, z. B. im Sportbereich, anzuknüpfen. Für die Zielgruppe der Multilokalen wäre ein zeitlich und räumlich flexibles Angebot nötig. Eine Idee eines Multilokalen ist die Etablierung kurzfristiger Trainingszeiten für Betriebssport, vor allem in den Sommermonaten, auf beziehungsweise in öffentlichen, kommunalen Sportstätten. Flexible und thematisch offene oder wechselnde Angebote tragen zudem zu einer Erweiterung der (bisherigen) Angebote bei, die gegebenenfalls auch von der monolokalen Bevölkerung genutzt werden könnten.

Trotz eines engen Zeitbudgets haben die Multilokalen Bekanntschaften an mehreren Orten und somit neue Netzwerke geschaffen, die sie teilweise auch nach der Aufgabe ihrer beruflichen Tätigkeit an den Orten nutzen. Hiervon können nicht nur die einzelnen Personen selbst, sondern auch die ländlich geprägten Kommunen profitieren. Denn sollte es den Kommunen gelingen, durch Angebote, etwa im Freizeitbereich, attraktiv genug für die Multilokalen zu sein, kommen diese öfter zu Besuch und verbringen ihre Freizeit oder ihren Urlaub an den Orten, wodurch der Einzelhandel und Tourismus (zumindest) profitieren können.

\subsubsection{Betriebliche Gestaltungsmöglichkeiten}

Ursache für ein Leben an mehreren Orten sind zumeist Projektarbeiten, Dienstreisen und Delegationen. Die Interviews mit den mehrörtig lebenden Beschäftigten machen deutlich, dass ihre Lebensweise in den meisten Fällen nur möglich ist, weil die Betriebe entsprechende Strukturen und Rahmenbedingungen, wie beispielsweise flexible Arbeitszeiten oder Unterstützungsleistungen wie Dienstwagen etc., schaffen. Diese oft freiwilligen (Zusatz-)Leistungen der Unternehmen können ausschlaggebend für Zuziehende, Multilokale und junge Menschen sein, um phasenweise in die Region zu kommen oder auch komplett dorthin zu ziehen. Wenn folglich die Betriebe interessante Unterstützungsangebote etablieren und ausweiten, können Fachkräftemangel oder die Landflucht junger Menschen teilweise kompensiert werden. Nichtsdestotrotz lassen sich nicht alle Berufe flexibilisieren. Einige Multilokale haben stark spezialisierte Tätigkeiten, die beispielsweise in Heimarbeit nicht verrichtet werden können, weil sie an Maschinen stattfinden müssen.

Obwohl die Interviewpartnerinnen/Interviewpartner häufig trotz Projektarbeiten und kurzen, befristeten Vertragslaufzeiten über längere Zeiträume an mehreren Orten leben, kann es für die Betriebe eine Herausforderung sein, weil die Beschäftigten eher einen Beschäftigungswechsel anstreben, z. B. aufgrund familiärer Verpflichtungen (z. B. Kindererziehung oder Pflege von Angehörigen). Hier können die Betriebe in einigen Fällen reagieren und befristete Arbeitsverhältnisse verringern oder gar abschaffen, um ihre multilokalen Beschäftigten zu Zuziehenden beziehungsweise monolokalen Beschäftigten zu machen. Unbefristete Arbeitsverhältnisse können vermutlich die mehrörtigen Lebensweisen verringern, weil die Menschen dann eher bereit sind, sich räumlich umzuorientieren. Aus wirtschaftlicher Sicht birgt dies ein Risiko, allerdings können so personelle Sicherheiten geschaffen werden. Sollten Entfristungen jedoch nicht möglich sein, könnten die Unternehmen ausreichend attraktive andere Optionen für die Beschäftigten schaffen. Dazu zählen aus der Sicht der Beschäftigten an erster Stelle finanzielle Anreize, aber auch Unterstützungsleistungen, z. B. in Form von Beratungsangeboten für die Wohnungssuche oder Freizeitangebote. Besonders in ländlich geprägten Räumen kann auch die Bereitstellung von temporärem Wohnraum seitens der Unternehmen für Multilokale sinnvoll sein, weil diese oft nicht schnell genug passenden Wohnraum finden (Greinke/Lange/Othengrafen 2018: 16). 


\subsubsection{Handlungsfelder für Regionen}

Die interviewten Multilokalen haben oft erst spät passenden Wohnraum (klein, möbliert und zentral) finden können. Denkbar ist, dass neben Vermittlungen von Wohnraum durch Betriebe auch Kommunen diese Aufgabe übernehmen und Wohnungsangebote bündeln (vgl. Greinke/Lange/Othengrafen 2018). Zudem kennen sich die Multilokalen untereinander nicht, obwohl sie teilweise ähnliche oder sogar dieselben Herkunftsorte haben. Hier könnten die Betriebe und die Kommunen ansetzen und entsprechende Angebote beispielsweise in Form von „Schwarzen Brettern" zur Vermittlung von Mitfahrgelegenheiten oder die Organisation von Vernetzungsveranstaltungen für die Zielgruppe Multilokale schaffen.

Durch diese Vernetzung könnten Regionen zudem im Bereich der Freizeitinfrastrukturen profitieren. Bislang vernetzen sich die mehrörtig Lebenden zwar innerhalb des Unternehmens mit der Kollegenschaft, aber es fehlt an einer übergreifenden Verknüpfung der Interessen. Obwohl den Multilokalen häufig nicht viel Zeit neben der Dienstzeit bleibt, sind sie an gemeinsamer Aktivität interessiert, sodass bestehende Formate besser ausgelastet oder neue Angebote geschaffen werden könnten. Durch die aktive Teilnahme an diesen Freizeittätigkeiten besteht zudem die Chance, dass die mehrörtig Lebenden sich in die Gemeinschaft integrieren und sogar ihre Familie mit an den Arbeitsort bringen, wodurch Anreize geschaffen werden können, sogar monolokal im Landkreis Diepholz leben zu wollen.

Aufgrund bisheriger Befunde aus der Forschung (Greinke/Lange/Othengrafen 2017), ist davon auszugehen, dass es für ländliche Kommunen, die potenziell von Multilokalität betroffen sind, vorteilhaft ist, wenn sie sich frühzeitig mit diesem Phänomen auseinandersetzen und Ideen und Ansätze entwickeln, wie sie multilokal Lebende in ihrer Kommune begegnen wollen. Wie können sie phasenweise Anwesende in Gemeinschaften einbringen? Welche Infrastrukturen müssen zukünftig entwickelt werden? Welcher Wohnraum wird nachgefragt?

\section{Fazit und Ausblick}

Dieser Beitrag macht deutlich, dass mehrörtige Lebensweisen in ländlichen Räumen ein bislang zu wenig betrachtetes Phänomen sind, welches durchaus Auswirkungen sowohl auf die betrieblichen als auch regionalen
Entwicklungen haben kann. Zwar stehen die untersuchten Kommunen bisher noch nicht vor der Herausforderung, mit einer großen Anzahl multilokal Lebender umgehen zu müssen, aber dies kann sich aufgrund der spezifischen Entwicklungen in der Arbeitswelt (und) in ländlichen Räumen mit zunehmendem Fachkräftemangel und abwandernder (junger) Bevölkerung verstärken.

Das Alltags- und Berufsleben in den temporär genutzten Räumen gestaltet sich innerhalb der Gruppe der Befragten häufig divers. Beeinflusst werden die multilokalen Lebensweisen zumeist durch betriebliche Rahmenbedingungen und Strukturen, die sie zum einen in eine mehrörtige Lebensweise drängen und zum anderen etwa aufgrund flexibler Arbeitszeiten erst ein Leben an mehreren Orten ermöglichen. Die Unternehmensstrukturen tragen folglich wesentlich dazu bei, wie die Menschen ihr Leben gestalten können (oder müssen). Denkbar ist, dass sich für Betriebe und Kommunen Potenziale ergeben, wie z. B. (phasenweiser) Zuzug von (Fach-)Arbeitskräften durch zielgruppenspezifische Wohnraumentwicklung, die durch die aufgezeigten Handlungsfelder für betriebliche und regionale Entwicklungen ausgeschöpft werden können.

Es ist davon auszugehen, dass sich sowohl Arbeitsals auch Lebenswelten und damit in Zusammenhang auch Formen multilokalen Lebens weiter wandeln und ausdifferenzieren werden. Vor allem neue Medien und Digitalisierungsprozesse können zukünftige temporär (physisch) genutzte Räume verändern. Denkbar ist, dass Dienstreisen nicht mehr so häufig nötig sind, weil Kommunikation (teilweise) digital stattfindet. Dadurch werden dann gegebenenfalls auch (dauerhafte oder phasenweise) Nebenorte weniger genutzt beziehungsweise frequentiert, weil Pendelhäufigkeiten weniger werden.

Desiderata ergeben sich hinsichtlich der (raumbezogenen) Theoriebildung in der Multilokalitätsforschung sowie belastbarer Aussagen zu Raumbeziehungen und der Quantifizierung des Phänomens. Zudem bleibt offen, wie Betriebe und Kommunen zukünftig auf mehrörtige Lebensweisen reagieren können und wollen. Und nicht zuletzt stellt sich die Frage, welche ökonomischen Implikationen die Zunahme und Ausdifferenzierung (berufsbedingter) multilokaler Lebensführungen mit sich bringen. Denn die multilokal Wohnenden sind an den jeweiligen Orten sowie unterwegs dazwischen auch Konsumentinnen/Konsumenten mit teilweise spezifischen Bedürfnissen nach Waren und Dienstleistungen. Daraus ergeben sich schon heute spezielle marktorientierte Angebote, insbesondere Infrastrukturangebote (z. B. Boardinghäuser, persönliche Lagerräume für aktuell nicht benö- 
tigte Gegenstände), Dienstleistungen zur Unterstützung der organisatorischen Anforderungen einer solchen Lebensweise (z. B. Housesitting, Begleitservices für allein reisende multilokal wohnende Kinder) sowie digitale Angebote in Form von Apps (z. B. zur Steuerung der Heizung aus der Ferne, zur Alltagsplanung innerhalb eines Haushalts, zur Überprüfung der Vitalfunktionen entfernt lebender Verwandter).

Gesellschaftlichen Entwicklungen wie die Multilokalität stellen Regionen und Gemeinden vor große Herausforderungen, denn sie sind oft nicht eindeutig, sondern widersprüchlich und verlaufen nicht linear. Daher braucht es auch neue Antworten: Gesucht sind solche, welche sich von langfristigem Denken und Planen verabschieden und stattdessen verschiedene mögliche Zukünfte so antizipieren, dass auch gegenüber Unerwartetem und Unvorhergesehenem eine passende Antwort möglich ist. So etwas Unerwartetes und Unvorhergesehenes sind und können an manchen Orten die Multilokalen mit ihrer spezifischen Lebensweise sein.

Danksagung: Danken möchten wir Prof. Dr. Rainer Danielzyk, Prof. Dr. Ingo Mose und Prof. Dr. Frank Othengrafen für die Betreuung der dieser Arbeit zugrunde liegenden Dissertation. Des Weiteren möchten wir uns beim Landkreis Diepholz und den interviewten Personen für die Bereitschaft zum Interview und ihre wertvollen Auskünfte bedanken. Dank gebührt außerdem der Akademie für Raumforschung und Landesplanung (ARL), die im Rahmen ihres Mentoring-Programms das gemeinsame Verfassen dieses Beitrags ermöglicht hat.

Funding information: Das Forschungsprojekt „Temporäre An- und Abwesenheiten und deren Auswirkungen auf Land und Gesellschaft" (www.tempaland.de) wird mit Mitteln des Bundesministeriums für Bildung und Forschung innerhalb der Fördermaßnahme „Kommunen innovativ" von 2016 bis 2019 gefördert.

The publication of this article was funded by the Open Access Fund of the Leibniz Universität Hannover.

\section{Literatur}

Albrow, M. (1997): Auf Reisen jenseits der Heimat. Soziale Landschaften in einer globalen Stadt. In: Beck, U. (Hrsg.): Kinder der Freiheit. Frankfurt am Main, 288-313.

Altrock, U.; Güntner, S.; Huning, S.; Nuissl, H.; Peters, D. (2005): Landliebe und Landleben. In: Planungsrundschau 12, 7-12.
ARL - Akademie für Raumforschung und Landesplanung (2016) Multilokale Lebensführung und räumliche Entwicklungen. Hannover. $=$ Positionspapier aus der ARL 104

Beck, U. (2008): Jenseits von Klasse und Nation. Individualisierung und Transnationalisierung sozialer Ungleichheiten. In: Soziale Welt 59, 4, 301-325.

BMEL - Bundesministerium für Ernährung und Landwirtschaft (2016): Bericht der Bundesregierung zur Entwicklung der ländlichen Räume 2016. Zweiter Bericht der Bundesregierung zur Entwicklung der ländlichen Räume. Berlin.

BMVBS-Bundesministerium fürVerkehr, Bau und Stadtentwicklung; BBSR - Bundesinstitut für Bau-, Stadt- und Raumforschung (Hrsg.) (2009): Ländliche Räume im demografischen Wandel. Berlin/Bonn. = BBSR-Online-Publikation 34/2009.

Brake, A. (2009): Photobasierte Befragung. In: Kühl, S. Strodtholz, P.; Taffertshofer, A. (Hrsg.): Handbuch Methoden der Organisationsforschung. Quantitative und Qualitative Methoden. Wiesbaden, 369-388. doi: 10.1007/978-3-53191570-8

Collmer, S.; Kümmel, G. (2005): Ein Job wie jeder andere? Zum Selbst- und Berufsverständnis von Soldaten. Baden-Baden. = Militär und Sozialwissenschaften 39.

Dannenberg, P.; Lang, T.; Lehmann, K. (2010): Einführung "Ländliche Räume“ in Deutschland: neuere Zugänge zu einer alten Kategorie. In: Europa Regional 18, 2-3, 55-59.

de Haan, L.; Zoomers, A. (2003): Development Geography at the Crossroads of Livelihood and Globalisation. In: Tijdschrift voor Economische en Sociale Geografie 94, 3, 350-362. doi: 10.1111/1467-9663.00262

Dick, E.; Reuschke, D. (2012): Multilocational Households in the Global South and North: Relevance, Features and Spatial Implications. In: Die Erde 143, 2, 177-194.

Dirksmeier, P. (2007): Mit Bourdieu gegen Bourdieu empirisch denken: Habitusanalyse mittels reflexiver Fotografie. In: ACME. An International Journal for Critical Geographies 6, 1, 73-97.

Dirksmeier, P. (2009): Urbanität als Habitus. Zur Sozialgeographie städtischen Lebens auf dem Land. Bielefeld.

Dittrich-Wesbuer, A. (2016): Multi-locality - New Challenges for Urban Development and Policies in Germany? In: TRIALOG - Zeitschrift für das Planen und Bauen im globalen Kontext 116/117, 1-2, 10-16.

Dittrich-Wesbuer, A.; Föbker, S. (2013): Multilokales Wohnen Verbreitung und Formen in Deutschland. In: Scheiner, J.; Blotevogel, H. H.; Frank, S.; Holz-Rau, C.; Schuster, N. (Hrsg.): Mobilitäten und Immobilitäten: Menschen - Ideen Dinge - Kulturen - Kapital. Essen, 391-402. = Dortmunder Beiträge zur Raumplanung 142.

Dittrich-Wesbuer, A.; Föbker, S.; Sturm, G. (2015): Multilokales Wohnen: Empirische Befunde zur Verbreitung in Deutschland. In: Weichhart, P.; Rumpolt, P. A. (Hrsg.): Mobil und doppelt sesshaft. Studien zur residenziellen Multilokalität. Wien, 121143. = Abhandlungen zur Geographie und Regionalforschung 18.

Dittrich-Wesbuer, A.; Plöger, J. (2013): Multilokalität und Transnationalität - Neue Herausforderungen für Stadtentwicklung und Stadtpolitik. In: Raumforschung und Raumordnung 71, 3, 195-205. doi: 10.1007/s13147-013-0237-8 
Fuhrer, U.; Kaiser, F. G. (1994): Multilokales Wohnen. Psychologische Aspekte der Freizeitmobilität. Bern.

Gallent, N.; Tewdwr-Jones, M. (2000): Rural Second Homes in Europe. Examining Housing Supply and Planning Control. Aldershot.

Greinke, L.; Lange, L.; Othengrafen, F. (2017): Multilokale Lebensweisen und ländliche Räume. Chance oder Herausforderung für Kommunen? In: RaumPlanung 192, 4, 14-21.

Greinke, L.; Lange, L.; Othengrafen, F. (2018): Multilokales Wohnen in ländlichen Räumen - Chancen und Herausforderungen. In: Planerln 18, 3, 44-46.

Hall, C. M.; Müller, D. K. (Hrsg.) (2004): Tourism, Mobility and Second Homes. Between Elite Landscape and Common Ground. Clevedon.

Helfferich, C. (2011): Die Qualität qualitativer Daten. Manual für die Durchführung qualitativer Interviews. Wiesbaden. doi: 10.1007/978-3-531-92076-4

Hesse, M.; Scheiner, J. (2007): Räumliche Mobilität im Kontext des sozialen Wandels: eine Typologie multilokalen Wohnens. In: Geographische Zeitschrift 95, 3, 138-154.

Hilti, N. (2009): Multilokales Wohnen: Bewegungen und Verortungen. In: Informationen zur Raumentwicklung 1/2, 77-86.

Hilti, N. (2013): Lebenswelten multilokal Wohnender. Eine Betrachtung des Spannungsfeldes von Bewegung und Verankerung. Wiesbaden. $=$ Stadt, Raum und Gesellschaft 25. doi: 10.1007/978-3-658-01046-1

Huchler, N. (2013): Wir Piloten. Navigation durch die fluide Arbeitswelt. Berlin.

Jurczyk, K.; Schier, M.; Szymenderski, P.; Lange, A.; Voß, G. G. (2009): Entgrenzte Arbeit - Entgrenzte Familie. Grenzmanagement im Alltag als neue Herausforderung. Berlin. = Forschung aus der Hans-Böckler-Stiftung 100.

Kaufmann, V. (2002): Re-thinking Mobility. Contemporary Sociology. Aldershot.

Krisch, R. (2002): Methoden einer sozialräumlichen Lebensweltanalyse. In: Deinet, U.; Krisch, R. (Hrsg.): Der sozialräumliche Blick der Jugendarbeit. Methoden und Bausteine zur Konzeptentwicklung und Qualifizierung. Opladen, 87-154.

Landkreis Diepholz (2016): Wohnraumversorgungskonzept Landkreis Diepholz. Diepholz.

Lange, L. (2018): Leben an mehreren Orten. Multilokalität und bürgerschaftliches Engagement in ländlich geprägten Räumen Niedersachsens. Berlin. = Ländliche Räume. Beiträge zur lokalen und regionalen Entwicklung 4.

Larsen, J.; Axhausen, K. W.; Urry, J. (2006): Geographies of Social Networks: Meetings, Travel and Communications. In: Mobilities 1, 2, 261-283. doi: 10.1080/17450100600726654

Leubert, N. (2013): Heute hier, morgen dort. Chancen und Herausforderungen multilokaler Lebensstile in Wolfsburg. Berlin. = Graue Reihe des Instituts für Stadt- und Regionalplanung 47.

Mayer, H. O. (2013): Interview und schriftliche Befragung. Grundlagen und Methoden empirischer Sozialforschung. München.

Mayring, P. (2010): Qualitative Inhaltsanalyse: Grundlagen und Techniken. Weinheim.
Meier, L.; Frank, S. (2016): Dwelling in mobile times: places, practices and contestations. In: Cultural Studies 30, 3, 362375. doi: 10.1080/09502386.2015.1113630

Meuser, M.; Nagel, U. (2002): ExpertInneninterviews - vielfach erprobt, wenig bedacht. Ein Beitrag zur qualitativen Methodendiskussion. In: Bogner, A.; Littig, B.; Menz, W. (Hrsg.): Das Experteninterview. Theorie, Methode, Anwendung. Opladen, 71-93.

Nadler, R. (2014): Plug\&Play Places. Lifeworlds of Multilocal Creative Knowledge Workers. Warschau/Berlin.

NHB - Niedersächsischer Heimatbund; ALR - Niedersächsische Akademie ländlicher Raum (2014): Zur Zukunft der Dörfer in Niedersachsen. Positionspapier des Niedersächsischen Heimatbundes e.V. und der Niedersächsischen Akademie ländlicher Raum e.V. zu Leitlinien zukünftiger Dorfentwicklung. Hannover.

Odermatt, A. (1990): Zweitwohnungen in Städten. Eine Untersuchung über die Zweitwohnungsproblematik in den fünf schweizerischen Grossstädten. Diplomarbeit an der Universität Zürich.

Peer, V. (2013): Was können ländliche Räume Hochqualifizierten bieten? Eine raumwissenschaftliche Analyse der Pull- und Push-Faktoren auf das Wanderungs- und Bleibeverhalten von FH-AbsolventInnen in ländlichen Regionen Österreichs. In: Ländlicher Raum 3, 1-11.

Petzold, K. (2013): Multilokalität als Handlungssituation. Lokale Identifikation, Kosmopolitismus und ortsbezogenes Handeln unter Mobilitätsbedingungen. Wiesbaden. doi: 10.1007/978-3531-19490-5

Pries, L. (1998): „Transmigranten“ als ein Typ von Arbeitswanderern in pluri-lokalen sozialen Räumen. In: Soziale Welt 49, 2, 135150.

Reuschke, D. (2010): Multilokales Wohnen. Raum-zeitliche Muster multilokaler Wohnarrangements von Shuttles und Personen in einer Fernbeziehung. Wiesbaden. doi: 10.1007/978-3-53192283-6

Rolshoven, J. (2007): Multilokalität als Lebensweise in der Spätmoderne. In: Schweizerisches Archiv für Volkskunde 103, 2, 157-179.

Saxinger, G. (2016): Unterwegs. Mobiles Leben in der Erdgas- und Erdölindustrie in Russlands Arktis. Wien.

Schier, M. (2009): Räumliche Entgrenzung von Arbeit und Familie. Die Herstellung von Familie unter Bedingungen von Multilokalität. In: Informationen zur Raumentwicklung 1/2, $55-66$

Schier, M. (2010): Multilokaler Alltag erwerbstätiger Eltern. Erweiterte Optionen oder erhöhte Probleme für die Lebensführung? In: Soeffner, H.-G. (Hrsg.): Unsichere Zeiten. Herausforderungen gesellschaftlicher Transformationen. Verhandlungen des 34 . Kongresses der Deutschen Gesellschaft für Soziologie in Jena 2008. CD-Rom. Wiesbaden.

Schier, M.; Hilti, N.; Schad, H.; Tippel, C.; Dittrich-Wesbuer, A.; Monz, A. (2015): Residential Multi-Locality Studies - The Added Value for Research on Families and Second Homes. In: Tijdschrift voor Economische en Sociale Geografie 106, 4, 439-452. doi: 10.1111/tesg.12155

Schmidt-Kallert, E. (2009): A New Paradigm of Urban Transition: Tracing the Livelihood Strategies of Multi-Locational Households. In: Die Erde 140, 3, 319-336. 
Schneider, N. F.; Limmer, R.; Ruckdeschel, K. (2002): Mobil, flexibel, gebunden. Familie und Beruf in der mobilen Gesellschaft. Frankfurt am Main/New York.

Seebacher, M. M. (2013): Die Praxis multilokalen Wohnens. Ein Leben an mehreren Orten - Habitus, Aktanten und Netzwerke. Wien. Masterarbeit an der Universität Wien.

Sheller, M. (2011): Mobility. In: Sociopedia.isa. doi: $10.1177 / 205684601163$

Steinbrink, M. (2009): Leben zwischen Land und Stadt. Migration, Translokalität und Verwundbarkeit in Südafrika. Wiesbaden. doi: 10.1007/978-3-531-91494-7

Sturm, G.; Meyer, K. (2009): Was können die Melderegister deutscher Großstädte zur Analyse von residenzieller Multilokalität beitragen? In: Informationen zur Raumentwicklung 1/2, 15-30.

Urry, J. (2007): Mobilities. Cambridge.

Viry, G.; Kaufmann, V.; Widmer, E. (2008): Switzerland - Mobility: a Life Stage Issue? In: Schneider, N. F.; Meil, G. (Hrsg.): Mobile Living Across Europe I. Relevance and Diversity of JobRelated Spatial Mobility in Six European Countries. Opladen/ Farmington Hills, 189-228.

Weichhart, P. (2009): Multilokalität - Konzepte, Theoriebezüge und Forschungsfragen. In: Informationen zur Raumentwicklung $1 / 2,1-14$.

Weichhart, P. (2015): Multi-local Living Arrangements - Terminology Issues. In: Weichhart, P.; Rumpolt, P.A. (Hrsg.): Mobil und doppelt sesshaft. Studien zur residenziellen Multilokalität. Wien, 61-82. = Abhandlungen zur Geographie und Regionalforschung 18.

Weichhart, P.; Rumpolt, P. A. (2015): Residenzielle Multilokalität. Problemlagen und Desiderata der Forschung. In: Weichhart, P.; Rumpolt, P. A. (Hrsg.): Mobil und doppelt sesshaft. Studien zur residenziellen Multilokalität. Wien, 11-60. = Abhandlungen zur Geographie und Regionalforschung 18.

Weiske, C.; Petzold, K.; Zierold, D. (2009): Multilokale Haushaltstypen. Bericht aus dem DFG-Projekt „Neue multilokale Haushaltstypen“ (2006-2008). In: Informationen zur Raumentwicklung 1/2, 67-75.

Wood, G.; Hilti, N.; Kramer, C.; Schier, M. (2015): A Residential Perspective on Multi-Locality. In: Tijdschrift voor Economische en Sociale Geografie 106, 4, 363-377. doi: 10.1111/tesg.12158 